\title{
A DIFFERENTIAL DELAY EQUATION ARISING FROM THE SIEVE OF ERATOSTHENES
}

\author{
A. Y. CHEER AND D. A. GOLDSTON
}

\begin{abstract}
The differential delay equation defined by $\omega(u)=1 / u$ for $1 \leq u \leq$ 2 and $(u \omega(u))^{\prime}=\omega(u-1)$ for $u \geq 2$ was introduced by Buchstab in connection with an asymptotic formula for the number of uncanceled terms in the sieve of Eratosthenes. Maier has recently used this result to show there is unexpected irregularity in the distribution of primes in short intervals. The function $\omega(u)$ is studied in this paper using numerical and analytical techniques. The results are applied to give some numerical constants in Maier's theorem.
\end{abstract}

\section{INTRODUCTION}

In the traditional sieve of Eratosthenes all the integers $1 \leq n \leq x$ which are multiples of the numbers $2 \leq m<x^{1 / 2}$ are removed. What remains after this process is the number 1 and all the prime numbers $p$ in the range $x^{1 / 2} \leq p \leq x$. By the prime number theorem there are asymptotically $x / \log x$ such numbers as $x \rightarrow \infty$. Suppose that only the numbers $2 \leq m<x^{1 / u}$, for $u \geq 2$, are sieved. In this case, not only prime numbers, but also numbers with all their prime factors larger than $x^{1 / u}$ are unsieved. It is natural in this case to ask for an asymptotic formula for the number of elements left unsieved. Buchstab [1] obtained such a formula. To state his result, define $\phi(x, y)$ to be the number of positive integers $\leq x$ with no prime factors $<y$, and further define the function $\omega(u)$ for $u \geq 1$ by the differential delay equation

$$
\begin{aligned}
\omega(u) & =\frac{1}{u}, \quad 1 \leq u \leq 2, \\
\frac{d}{d u}(u \omega(u)) & =\omega(u-1), \quad u \geq 2,
\end{aligned}
$$

where in ( $1 \mathrm{~b})$ the right-hand derivative of $\omega(u)$ is taken at $u=2$. Buchstab proved, for $x \rightarrow \infty$, that

$$
\phi(x, y) \sim x e^{\gamma^{\prime}} \omega(u) \prod_{p<y}\left(1-\frac{1}{p}\right),
$$

Received April 29, 1988; revised March 31, 1989.

1980 Mathematics Subject Classification (1985 Revision). Primary 11 N35.

The first author's research was supported in part by Office of Research and Professional Development Award U.C. Davis, and by NASA-Ames Grant NAC2-172.

The second author's research was supported in part by NSF Grant DMS8705710. 
where $y=x^{1 / u}$, and $u \geq 1$ is fixed. By Mertens's formula we have

$$
\prod_{p<y}\left(1-\frac{1}{p}\right) \sim \frac{e^{-\gamma}}{\log y},
$$

which implies that (2) may be reformulated as

$$
\phi(x, y) \sim u \omega(u) \frac{x}{\log x} .
$$

This result of Buchstab is interesting because it shows that $\phi(x, y)$ is a somewhat irregular function. The factor $x \prod_{p<y}\left(1-\frac{1}{p}\right)$ is the "expected value" of $\phi(x, y)$, and for $y \ll \log x$ one finds by the Legendre sieve [4, p. 200] that $\phi(x, y)$ is asymptotic to this expected value. However, for larger sieving ranges, equation (2) shows that $\phi(x, y)$ oscillates from this expected value by the factor $e^{y} \omega(u)$. In view of this, it is important to study the function $\omega(u)$.

It has been shown by Buchstab [1], de Bruijn [2], and Hua [5] that $\omega(u) \sim e^{-\gamma}$ as $u \rightarrow \infty$, and further that $\omega(u)$ converges faster than exponentially to $e^{-\gamma}$. The best result is due to Hua, who proved that

$$
\left|\omega(u)-e^{-\gamma}\right| \leq e^{-u(\log u+\log \log u+(\log \log u / \log u)-1)+O(u / \log u)} .
$$

A surprising application of Buchstab's result has recently been made by Maier [6]. Using an ingenious construction, Maier proved that the number of primes in short intervals $\left[x, x+(\log x)^{C}\right], C>1$, is sometimes larger than, and sometimes smaller than the expected number $(\log x)^{C-1}$. To state his result, let

$$
M_{+}(v)=\max _{u \geq v}\left(\omega(u)-e^{-\gamma}\right), \quad M_{-}(v)=\min _{u \geq v}\left(\omega(u)-e^{-\gamma}\right) .
$$

We will see later that these functions are well defined. Let $\pi(x)$ denote the number of primes $\leq x$. Maier proved, for any fixed $C>1$,

$$
\begin{aligned}
& \limsup _{x \rightarrow \infty} \frac{\pi\left(x+(\log x)^{C}\right)-\pi(x)}{(\log x)^{C-1}} \geq 1+e^{\gamma} M_{+}(C) \\
& \liminf _{x \rightarrow \infty} \frac{\pi\left(x+(\log x)^{C}\right)-\pi(x)}{(\log x)^{C-1}} \leq 1+e^{\gamma} M_{-}(C) .
\end{aligned}
$$

Furthermore, using a method involving the adjoint equation of (1), due to de Bruijn, Maier showed that $\omega(u)-e^{-\gamma}$ changes sign in every interval of length one. Hence, for all $C \geq 1$,

$$
e^{\gamma} M_{+}(C)>0, \quad e^{\gamma} M_{-}(C)<0 .
$$

In this paper we compute $\omega(u)-e^{-\gamma}$ numerically for small values of $u$ and thus provide some numerical constants for Maier's results. We also prove some new results on $\omega(u)$. We start by proving a theorem on $M_{+}(v)$ and $M_{-}(v)$ for the function $\omega(u)$. 
Theorem 1. For $v \geq 2$, we have

$$
M_{+}(v)=\max _{v \leq u \leq v+2}\left(\omega(u)-e^{-\gamma}\right), \quad M_{-}(v)=\min _{v \leq u \leq v+2}\left(\omega(u)-e^{-\gamma}\right) .
$$

Theorem 1 indicates that the maxima and minima of $\omega(u)-e^{-\gamma}$ get smaller in intervals of length greater than 2 . If we examine $\omega(u)-e^{-\gamma}$ more closely, we find it has a regular oscillatory pattern. Let us denote the zeros of $\omega(u)-e^{-\gamma}$ in increasing size by $\lambda_{1}, \lambda_{2}, \lambda_{3}, \ldots$. Except for the relative minimum at $u=2$ which is a cusp, the relative maxima and minima of $\omega(u)-e^{-\gamma}$ occur at the critical points where $\omega^{\prime}(u)=0$. We let $c_{1}=2$ and denote the critical points in increasing size by $c_{2}, c_{3}, \ldots$. As mentioned before, for every $u \geq 2$, the interval $(u, u+1)$ contains a zero $\lambda_{i}$, and it is easy to prove that it will also contain a critical point $c_{j}($ see $\S 2)$. We add to this information the following result:

Theorem 2. Each interval $[u, u+1]$ contains at most two zeros for $u \geq 2$, and at most two critical points. Furthermore, we have $\lambda_{1}<c_{1}<\lambda_{2}<\cdots$; the $c_{2 k}$ are relative maxima with $\omega\left(c_{2 k}\right)-e^{-\gamma}>0$, and the $c_{2 k-1}$ are relative minima with $\omega\left(c_{2 k-1}\right)-e^{-\gamma}<0$.

It is easily proved that the interval $\left[\lambda_{k}, \lambda_{k}+1\right]$ always contains two critical points, while $\left[c_{k}-1, c_{k}\right]$ always contains two zeros.

We prove these theorems in $\S 2$. In $\S 3$ we provide numerical results on $\omega(u)$ for $1 \leq u \leq 11$. These results are obtained by solving (1) iteratively using power series solutions. A similar procedure has been discussed in [3].

We expect that as $n \rightarrow \infty, \lambda_{n+1}-\lambda_{n} \rightarrow 1, c_{n+1}-c_{n} \rightarrow 1$, and $c_{n}-\lambda_{n} \rightarrow 0$. However the convergence is not very rapid. These results are summarized in Table 2. [Added in proof: These results have now been proved by A. Hildebrand.]

\section{PROOF OF THE THEOREMS}

Suppose $u>2$, and let

$$
W(u)=\omega(u)-e^{-\gamma} .
$$

By (lb) we see that $W(u)$ satisfies

$$
u W^{\prime}(u)=W(u-1)-W(u)
$$

or

$$
\frac{d}{d u}(u W(u))=W(u-1)
$$

We now claim that there is a value $c$ in every interval $(u, u+1)$ such that $W^{\prime}(c)=0$. Note first that $(10)$ implies

$$
u W^{\prime}(u)=-\int_{u-1}^{u} W^{\prime}(t) d t .
$$


Suppose that $W^{\prime}(t) \neq 0$ in $(u-1, u)$. Then $W^{\prime}(t)$ is either always positive or always negative in this interval, since $W^{\prime}(t)$ is continuous for $t>2$. Suppose $W^{\prime}(t)$ is positive in $(u-1, u)$. Then by $(12), W^{\prime}(u)<0$, a contradiction to the continuity of $W^{\prime}(t)$. The same argument applies if $W^{\prime}(t)$ is negative. If $W(t) \equiv 0$ in this interval, then this will imply $W(t) \equiv 0$ for all $t \leq u$ contradicting the value of $W(u)$ in the initial range $1 \leq u \leq 2$. Thus, there is a sign change in $(u-1, u)$.

The same result also holds for $W(u)$ (i.e., every interval $(u, u+1)$ contains a zero of $W(u))$ because (see [5]), for $u \geq 2$,

$$
u h(u-1) W(u)=-\int_{u-1}^{u} W(t) h(t) d t
$$

where

$$
h(u)=\int_{0}^{\infty} \exp \left(-u x-x-\int_{0}^{x} \frac{1-e^{-t}}{t} d t\right) d x
$$

and $h(u)>0$ is a decreasing function, with $h(u) \sim 1 / u$ as $u \rightarrow \infty$. Using (13) and the positivity of $h(u)$, we find by the same argument just used that every interval $(u, u+1)$ has a zero $\lambda_{k}$. With this preparation we can now prove the theorems.

Proof of Theorem 1. To prove the result for $M_{+}(v)$, it suffices to prove that given a positive relative maximum at $c, c \geq 3$, there will be a value $d^{\prime}, c-2 \leq$ $d^{\prime} \leq c$, such that $W\left(d^{\prime}\right)>W(c)$. By (13) and the mean value theorem for integrals there is a value $d$ such that $c-1 \leq d \leq c$ and

$$
W(d) h(d)=-\operatorname{ch}(c-1) W(c) .
$$

Applying (13) to $W(d)$ again, we find a number $d^{\prime}, d-1 \leq d^{\prime} \leq d$, such that

$$
W\left(d^{\prime}\right) h\left(d^{\prime}\right)=-d h(d-1) W(d),
$$

and hence

$$
W\left(d^{\prime}\right)=\frac{c d h(c-1) h(d-1) W(c)}{h(d) h\left(d^{\prime}\right)} \geq c d W(c),
$$

since $h(u)$ is positive and decreasing. This proves the result for $v \geq c-2$. The first maximum is at $c_{2}=2.7632 \ldots$ and is positive, and the second is at $c_{4}=4.2175 \ldots$ and is also positive (see Table 1 and Figure $1 \mathrm{~b}$ ). Therefore, our proof shows that the result holds for $v \geq c_{4}-2$. But we have $M_{+}(v)=$ $\max _{v \leq u \leq c_{2}}\left(\omega(u)-e^{-\gamma}\right)$ for $1 \leq v \leq c_{2}$, and since $c_{4}-2<c_{2}$, the result holds for all $v \geq 1$. The proof for $M_{-}(v)$ is similar.

Proof of Theorem 2. First note that (10) implies immediately that at critical points $c_{k}>2$ we have

$$
W\left(c_{k}-1\right)=W\left(c_{k}\right) .
$$




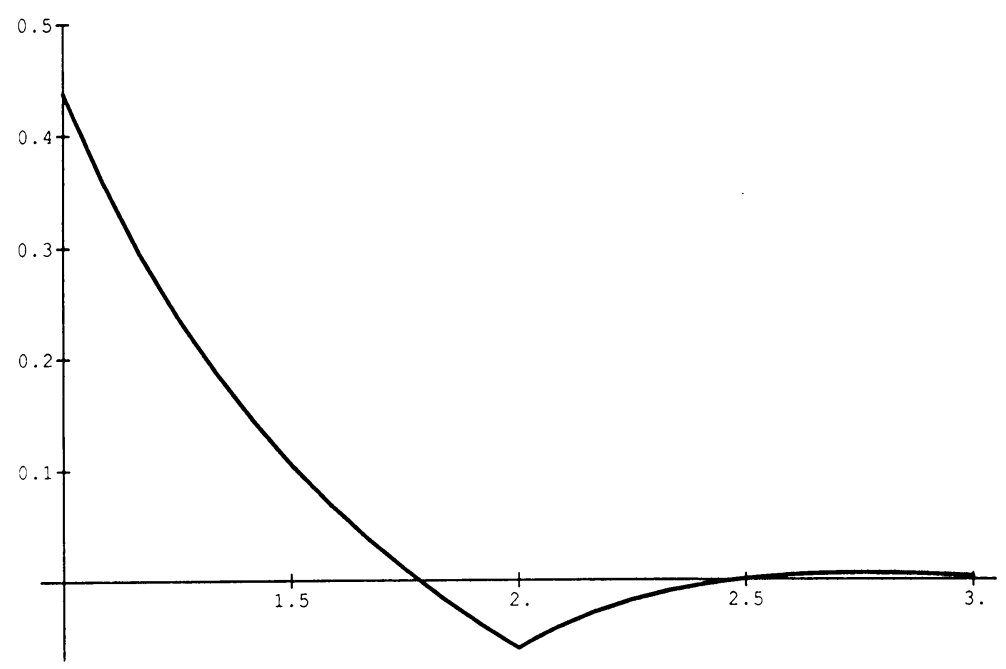

Figure 1a. $W(u)$ for $1 \leq u \leq 3$.

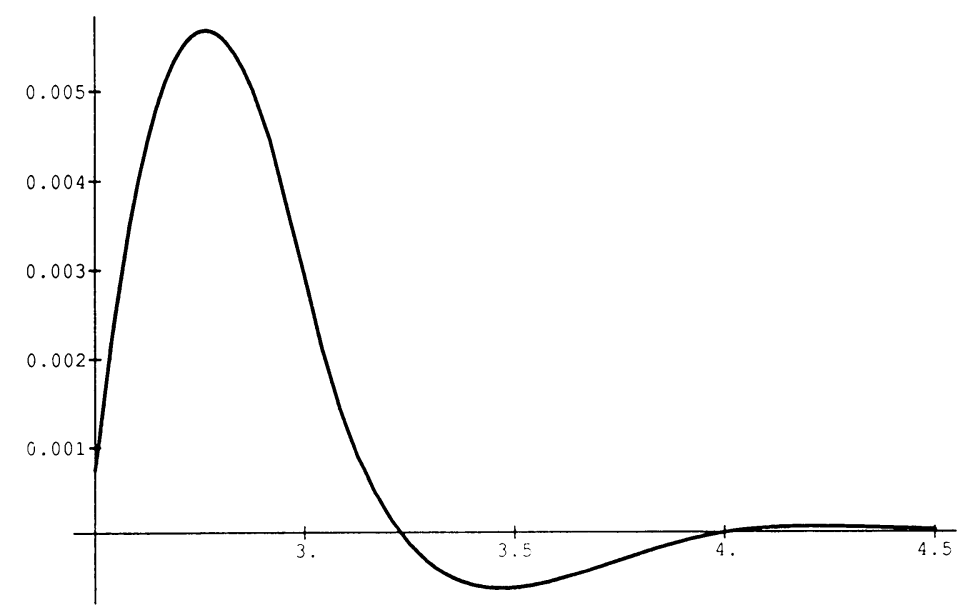

Figure 1b. $\quad W(u)$ for $2.5 \leq u \leq 4.5$.

Our method for proving Theorem 2 is by induction. We note that the theorem is true initially for $u \leq 3$, as may be easily verified since $\omega(u)=(\log (u-1)+1) / u$ for $2 \leq u \leq 3$ (see also Figure 1a). Now suppose the theorem is true up to $c_{k}$, a positive maximum of $W(u)$, and consider the interval $\left[c_{k}-1, c_{k}\right]$ for $k \geq 2$. Let us suppose further that $W(u)$ is as indicated in Figure 2, i.e., $W(u)$ decreases in $\left(c_{k}-1, c_{k-1}\right)$, has a negative minimum at $c_{k-1}$, and then increases in $\left(c_{k-1}, c_{k}\right)$, and hence has precisely two zeros $\lambda_{k-1}, \lambda_{k}$ in the interval. Further suppose that the only critical points in $\left[c_{k}-1, c_{k}\right]$ are the relative minimum at $c_{k-1}$ and the relative maximum at $c_{k}$ (if $k=2$ the relative minimum $c_{1}$ 


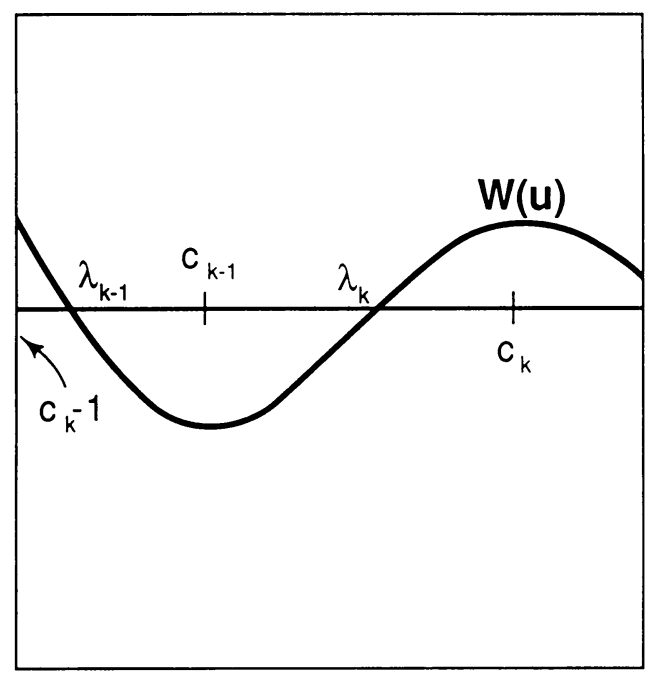

FIGURE 2

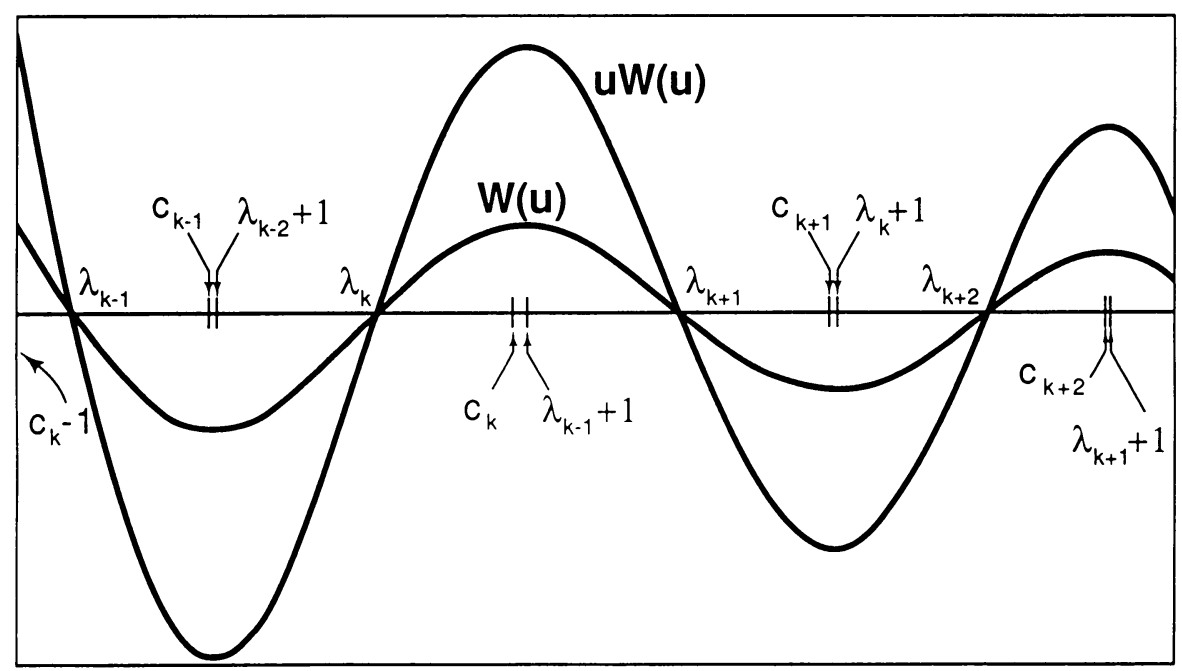

FIGURE 3

is a cusp, not a critical point). We will prove that $W(u)$ will duplicate this behavior in the next interval $\left[c_{k}, c_{k+2}\right]$, i.e., $W(u)$ will decrease, hit a zero at $\lambda_{k+1}$, continue to decrease to a negative minimum at $c_{k+1}$, then increase through a zero $\lambda_{k+2}$ to a positive maximum at $c_{k+2}$ (see Figure 3 ). The only critical points will be at $c_{k+1}$ and $c_{k+2}$. Further $c_{k+1}-c_{k-1}>1, c_{k+2}-c_{k}>$ $1, \lambda_{k+1}-\lambda_{k-1}>1$, and $\lambda_{k+2}-\lambda_{k}>1$. This will prove Theorem 2 for $u \leq c_{k+2}$. The above argument can now be applied to the next interval $\left[c_{k+2}-1, c_{k+2}\right]$. Hence, the theorem will hold by induction for all $u$. 
We first note that if $c$ is a critical point which is not a relative maximum or relative minimum, then $W^{\prime}(c)=W^{\prime \prime}(c)=0$. On differentiating (10), we conclude that $W^{\prime}(c-1)=0$. Thus, critical points that are inflection points can only occur at $u=c$ if $u=c-1$ is also a critical point. Since by assumption the only critical points in $\left[c_{k}-1, c_{k}\right]$ are at $c_{k-1}$ and $c_{k}$, the only possible critical points which are not extrema in $\left[c_{k}, c_{k}+1\right]$ are at $c_{k-1}+1$ or $c_{k}+1$. These cases will be treated later.

By equation (11) the sign of $W(u)$ in the interval $\left[c_{k}-1, c_{k}\right]$ determines whether $u W(u)$ increases or decreases in the interval $\left[c_{k}, c_{k}+1\right]$. Further, $u W(u)$ and $W(u)$ have the same zeros and the same sign. Thus $u W(u)$ is as pictured in Figure 3; i.e., $u W(u)$ has a zero at $u=\lambda_{k}, u W(u)>0$ for $\lambda_{k}<u \leq c_{k}, u W(u)$ increases for $c_{k}<u<\lambda_{k-1}+1$, and $u W(u)$ decreases for $\lambda_{k-1}+1<u<\lambda_{k}+1$. Since $W(u)$ has a zero in every open interval of length $1, W(u)$ must have a zero $\lambda_{k+1}$ in $\left(\lambda_{k}, \lambda_{k}+1\right)$, and hence $W(u)$ and $u W(u)$ have a unique zero at $\lambda_{k+1}$. Note $\lambda_{k+1}>\lambda_{k-1}+1$, so that $\lambda_{k+1}-\lambda_{k-1}>1$. Also $W\left(\lambda_{k}+1\right)<0$.

Next, $u W(u)$ will increase for $\lambda_{k}+1<u<\lambda_{k+1}+1$. Further, it must hit a unique zero at $\lambda_{k+2}$, since otherwise the interval $\left(\lambda_{k+1}, \lambda_{k+1}+1\right)$ would have no zero. Also $\lambda_{k+2}>\lambda_{k}+1$, so $\lambda_{k+2}-\lambda_{k}>1$, and $W\left(\lambda_{k+1}+1\right)>0$.

It remains to prove that $W(u)$ is as shown in Figure 3 , and $c_{k+1}-c_{k-1}>1$ and $c_{k+2}-c_{k}>1$. We begin by noting that if $u W(u)$ decreases and $W(u)>$ 0 in an interval, then $W(u)$ also decreases in that interval; and if $u W(u)$ increases and $W(u)<0$ in an interval, then $W(u)$ increases in that interval. Therefore, $W(u)$ decreases in $\left(\lambda_{k-1}+1, \lambda_{k+1}\right)$ and increases in $\left(\lambda_{k}+1, \lambda_{k+2}\right)$. Let $c_{k+1}$ be the next relative minimum of $W(u)$ for $u \geq \lambda_{k+1}$.

We now show $W(u)$ decreases for $c_{k}<u \leq \lambda_{k-1}+1$. To see this, let $\alpha$ and $\beta$ be any two numbers in this interval with $c_{k} \leq \alpha<\beta \leq \lambda_{k-1}+1$. On integrating (11), we have

$$
\beta W(\beta)-\alpha W(\alpha)=\int_{\alpha-1}^{\beta-1} W(t) d t<(\beta-\alpha) W(\alpha-1),
$$

since by Figure $2, W(t)$ is positive and decreasing in the interval $(\alpha-1, \beta-1) \subset$ $\left(c_{k}-1, \lambda_{k-1}\right)$. Hence we have

$$
\begin{aligned}
\beta(W(\beta)-W(\alpha)) & =\beta W(\beta)-\alpha W(\alpha)-(\beta-\alpha) W(\alpha) \\
& <(\beta-\alpha)(W(\alpha-1)-W(\alpha))=(\beta-\alpha) \alpha W^{\prime}(\alpha),
\end{aligned}
$$

which gives

$$
\alpha W^{\prime}(\alpha)>\frac{\beta}{\beta-\alpha}(W(\beta)-W(\alpha))
$$

From (18) we see $W(u)$ decreases in $\left(c_{k}, \lambda_{k-1}+1\right)$, since $W(u)$ initially decreases and if there were a value $\alpha$ where $W^{\prime}(\alpha)=0$, then (18) would imply $W(\beta)<W(\alpha)$ for all $\beta>\alpha$. Hence $\alpha$ would not be a relative minimum, and there are no critical points which are inflections in this interval. Furthermore, $W(u)>0$ in $\left(c_{k}, \lambda_{k-1}+1\right)$, because $u W(u)>0$ in this interval. 
Since $c_{k+1}$ is defined as the next relative minimum of $W(u)$ for $u \geq \lambda_{k+1}$, we have that $W(u)$ decreases in $\left(\lambda_{k+1}, c_{k+1}\right)$.

We next consider the interval $\left(c_{k+1}, \lambda_{k}+1\right)$. We note $c_{k+1} \neq \lambda_{k+1}$, since equality would imply $\lambda_{k+1}-1=\lambda_{k}$ or $\lambda_{k-1}$. The first case is impossible because this would imply the interval $\left(\lambda_{k}, \lambda_{k+1}\right)$ of length one has no zeros. The second case is impossible since $\lambda_{k+1}>\lambda_{k-1}+1$.

We now prove $c_{k-1}+1<c_{k+1}$. For if not, then either $c_{k+1}<c_{k-1}+1$, or $c_{k+1}=c_{k-1}+1$. In the first case let $c_{k+1}<\beta<c_{k-1}+1$. Then, since $W(t)$ is negative and decreasing in $\left(c_{k+1}-1, c_{k-1}\right)$,

$$
\begin{aligned}
\beta W(\beta)-c_{k+1} W\left(c_{k+1}\right) & =\int_{c_{k+1}-1}^{\beta-1} W(t) d t<\left(\beta-c_{k+1}\right) W\left(c_{k+1}-1\right) \\
& =\left(\beta-c_{k+1}\right) W\left(c_{k+1}\right),
\end{aligned}
$$

where we used (16) in the last line. Hence, $W(\beta)<W\left(c_{k+1}\right)$ for any $\beta>c_{k+1}$, contradicting the fact that $c_{k+1}$ is a relative minimum. In the case $c_{k+1}=$ $c_{k-1}+1$, we have $W\left(c_{k+1}\right)=W\left(c_{k-1}\right)$, and for $\lambda_{k+1}<\beta<c_{k+1}=c_{k-1}+1$,

$$
\begin{aligned}
-\beta W(\beta)+c_{k+1} W\left(c_{k+1}\right) & =\int_{\beta-1}^{c_{k+1}-1} W(t) d t>\left(c_{k+1}-\beta\right) W\left(c_{k+1}-1\right) \\
& =\left(c_{k+1}-\beta\right) W\left(c_{k+1}\right),
\end{aligned}
$$

implying $W(\beta)<W\left(c_{k+1}\right)$, which is impossible if $c_{k+1}$ is a relative minimum. This argument also shows that $c_{k-1}+1$ is not an inflection point as mentioned earlier.

Next, we prove that $W(u)$ increases in $\left(c_{k+1}, \lambda_{k}+1\right)$. Let $\alpha$ and $\beta$ be any numbers satisfying $c_{k+1}<\alpha<\beta<\lambda_{k}+1$. By the same argument used to prove (18), we have

$$
\alpha W^{\prime}(\alpha)<\frac{\beta}{\beta-\alpha}(W(\beta)-W(\alpha)) .
$$

If $W(u)$ did not increase through this interval, then there is a point $u=a$ in the interval where $W^{\prime}(a)=0$. Letting $\alpha=a$ implies $W(\beta)-W(\alpha)>0$ for any $\beta>\alpha$, which shows that $W(u)$ increases. Let $c_{k+2}$ be the next relative maximum of $W(u)$ for $u>\lambda_{k+2}$. Then $W(u)$ increases in $\left(\lambda_{k+2}, c_{k+2}\right)$. The proof that $c_{k+2}>c_{k}+1$ is the same as the previous argument that $c_{k+1}>$ $c_{k-1}+1$, which also shows that $c_{k}+1$ is not an inflection. This completes the proof of Theorem 2 .

\section{Numerical CALCUlations}

To compute $\omega(u)$ numerically, we start with a power series solution in the initial range and then iteratively obtain new power series solutions by substituting into ( $1 \mathrm{~b})$ and integrating term by term. Let

$$
\omega_{j}(u)=\omega(u) \quad \text { for } j \leq u \leq j+1 .
$$


Thus, $\omega_{1}(u)=1 / u$ for $1 \leq u \leq 2$, and by integrating (1),

$$
\omega_{2}(u)=\frac{\log (u-1)+1}{u} \quad \text { for } 2 \leq u \leq 3 .
$$

In general, for $j \geq 1$,

$$
u \omega_{j+1}(u)=\int_{j}^{u-1} \omega_{j}(t) d t+(j+1) \omega_{j}(j+1) \quad \text { for } j+1 \leq u \leq j+2
$$

We expand $\omega_{j}(u)$ into a power series about $u=j+1$ :

$$
\omega_{j}(u)=\sum_{k=0}^{\infty} a_{k}(j)(u-(j+1))^{k} \quad \text { for } j \leq u \leq j+1 .
$$

For $j=2$ we have

$$
\begin{aligned}
\omega_{2}(u) & =\frac{\log (u-1)+1}{u} \\
& =\left(\frac{1}{3+(u-3)}\right)(1+\log (2+(u-3))) \\
& =\left(\sum_{m=0}^{\infty} \frac{(-1)^{m}(u-3)^{m}}{3^{m+1}}\right)\left(1+\log 2+\sum_{n=1}^{\infty} \frac{(-1)^{n+1}(u-3)^{n}}{n 2^{n}}\right) \\
& =\sum_{k=0}^{\infty} a_{k}(2)(u-3)^{k},
\end{aligned}
$$

where $a_{0}(2)=(1+\log 2) / 3$, and for $k \geq 1$,

$$
\begin{aligned}
a_{k}(2) & =(-1)^{k+1}\left(-\frac{1+\log 2}{3^{k+1}}+\frac{1}{3\left(2^{k}\right)} \sum_{m=0}^{k-1} \frac{1}{(k-m)}\left(\frac{2}{3}\right)^{m}\right) \\
& =(-1)^{k+1} b_{k}(2), \quad \text { say. }
\end{aligned}
$$

We have $b_{1}(2)=-0.02146 \ldots$, and it is easily shown that $b_{k}(2) \geq 0$ for $k \geq 2$. Since

$$
\sum_{m=0}^{k-1} \frac{1}{(k-m)}\left(\frac{2}{3}\right)^{m} \leq \sum_{m=0}^{k-1}\left(\frac{2}{3}\right)^{m}=3-3\left(\frac{2}{3}\right)^{k}<3,
$$

we have

$$
b_{k}(2)<1 / 2^{k} \quad \text { for } k \geq 2 \text {. }
$$

An induction argument using (21) shows that in general, for $k \geq 1$ and $j \geq 2$,

$$
\left|a_{k}(j)\right|<1 / 2^{k} \text {. }
$$

This shows that the series solutions converge rapidly.

In computing $\omega_{j}(u)$, we begin by truncating the series for $\omega_{2}(u)$ and computing the coefficients of the resulting polynomial to a given accuracy. Let this approximation to $\omega_{2}(u)$ be denoted by $T_{2}(u)$, and put $E_{2}(u)=\omega_{2}(u)-T_{2}(u)$ 
and $E=\max _{2 \leq u \leq 3}\left|\omega_{2}(u)-T_{2}(u)\right|$. On substituting $T_{2}(u)$ into (21) we obtain, for $3 \leq u \leq 4$, a new series $T_{3}(u)$ given by

$$
\begin{aligned}
u T_{3}(u) & =\int_{2}^{u-1} T_{2}(v) d v+3 T_{2}(3) \\
& =\int_{2}^{u-1}\left(\omega_{2}(v)-E_{2}(v)\right) d v+3\left(\omega_{2}(3)-E_{2}(3)\right) \\
& =u \omega_{3}(u)-u E_{3}(u)
\end{aligned}
$$

where

$$
\left|E_{3}(u)\right|=\left|\frac{1}{u}\left(\int_{2}^{u-1} E_{2}(v) d v+3 E_{2}(3)\right)\right| \leq \frac{(u-3) E+3 E}{u} \leq E .
$$

The same argument clearly applies for each iteration, and thus, if we start with a given accuracy, we will retain it at each step, aside from round-off and other computational errors.

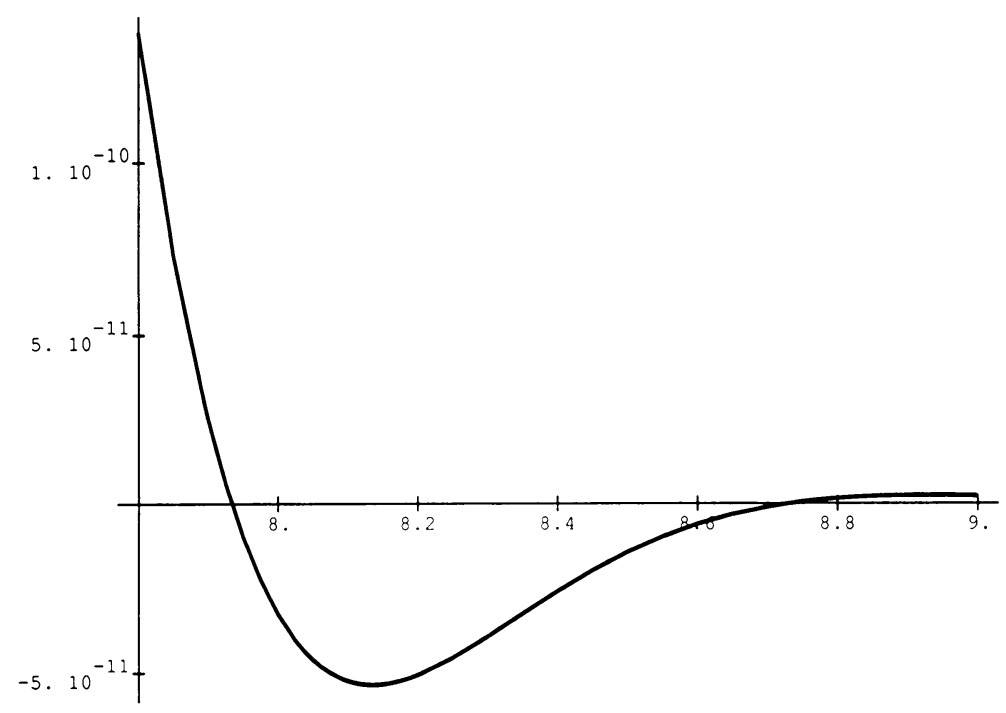

Figure 4. $W(u)=\omega(u)-e^{-\gamma}$ for $7.8 \leq u \leq 9$.

Our calculations were initially done using MACSYMA on a Vax 780. The series for $\omega_{2}(u)$ was truncated at 50 terms and the coefficients were accurate to 16 digits. This polynomial was then used with (21) to determine the approximations to $\omega_{j}(u)$. Later we used Mathematica on a Mac II to redo our calculations. We first used a series for $\omega_{2}(u)$ with 60 terms and did all calculations with 25-digit accuracy, and then repeated the calculations with 100 terms and 50-digit accuracy.

The error in truncating $\omega_{2}(u)$ after the $k$ th term is less than the absolute value of the next term since the series is alternating with decreasing coefficients. 
Hence, the error is at worst $1 / 2^{k+1}$. For the case $k=50$, the error is less than $2^{-51}=4.4 \times 10^{-16}$, while for $k=60$ and $k=100$ the errors are $2^{-61}=4.3 \times 10^{-19}$ and $2^{-101}=3.9 \times 10^{-31}$, respectively. The zeros and critical points of $\omega(u)$ were computed using Newton's method. Comparing the results from the different approximations provided a check on the accuracy of our calculations. The results in Tables 1 and 2 are in complete agreement between the calculations with $k=60$ and $k=100$, with the exception of the last digit in the value of $\omega\left(c_{12}\right)-e^{-\gamma}$, where one would expect the accuracy of the calculation for $k=60$ to be at most $10^{-19}$.

\section{TABLE 1}

\begin{tabular}{|clc|}
\hline Point & $u$ & $\omega(u)-e^{-\gamma}$ \\
\hline$\lambda_{1}$ & 1.78107 & 0 \\
$c_{1}$ & 2 & $-6.14594 \times 10^{-2}$ \\
$\lambda_{2}$ & 2.48332 & 0 \\
$c_{2}$ & 2.76322 & $5.68380 \times 10^{-3}$ \\
$\lambda_{3}$ & 3.22700 & 0 \\
$c_{3}$ & 3.46974 & $-6.36654 \times 10^{-4}$ \\
$\lambda_{4}$ & 4.00171 & 0 \\
$c_{4}$ & 4.21753 & $6.22072 \times 10^{-5}$ \\
$\lambda_{5}$ & 4.78578 & 0 \\
$c_{5}$ & 4.99493 & $-5.01722 \times 10^{-6}$ \\
$\lambda_{6}$ & 5.56650 & 0 \\
$c_{6}$ & 5.77973 & $3.38871 \times 10^{-7}$ \\
$\lambda_{7}$ & 6.35072 & 0 \\
$c_{7}$ & 6.56115 & $-2.03006 \times 10^{-8}$ \\
$\lambda_{8}$ & 7.14000 & 0 \\
$c_{8}$ & 7.34605 & $1.09487 \times 10^{-9}$ \\
$\lambda_{9}$ & 7.93400 & 0 \\
$c_{9}$ & 8.13590 & $-5.33852 \times 10^{-11}$ \\
$\lambda_{10}$ & 8.73170 & 0 \\
$c_{10}$ & 8.93036 & $2.36503 \times 10^{-12}$ \\
$\lambda_{11}$ & 9.53230 & 0 \\
$c_{11}$ & 9.72844 & $-9.58198 \times 10^{-14}$ \\
$\lambda_{12}$ & 10.33550 & 0 \\
$c_{12}$ & 10.52934 & $3.57568 \times 10^{-15}$ \\
& & \\
& &
\end{tabular}

The results of our calculations are indicated in Tables 1 and 2. Table 1 lists the zeros and critical points of $\omega(u)-e^{-\gamma}$, and the values of $\omega(u)-e^{-\gamma}$ at these critical points. These values are truncated at 5 digits. The size of Maier's constants $e^{\gamma} M_{+}(C)$ and $e^{\gamma} M_{-}(C)$ may be estimated from this table, since $M_{+}(C) \geq \omega\left(c_{j}\right)-e^{-\gamma}$ for any $c_{j} \geq C$, and $M_{-}(C) \leq \omega\left(c_{j}\right)-e^{-\gamma}$ for any $c_{j} \geq C$. In fact $M_{+}(C)=\omega\left(c_{j}\right)-e^{-\gamma}$ for even $j$ and $\lambda_{j-1} \leq C \leq c_{j}$, and $M_{-}(C)=\omega\left(c_{j}\right)-e^{-\gamma}$ for odd $j$ and $\lambda_{j-1} \leq C \leq c_{j}$. Thus, for example, there are arbitrarily large values of $x$ where the interval $\left[x, x+\log ^{8} x\right]$ 
TABLE 2

\begin{tabular}{|rcccc|}
\hline$n$ & $\lambda_{n+1}-\lambda_{n}$ & $c_{n+1}-c_{n}$ & $c_{n+1}-\lambda_{n}$ & $c_{n}-\lambda_{n}$ \\
\hline & .70225 & .76322 & .98215 & .21892 \\
1 & .70225 & .76322 & .98215 & .21892 \\
2 & .74367 & .70652 & .98642 & .27989 \\
3 & .77470 & .74779 & .99053 & .24274 \\
4 & .78407 & .77739 & .99322 & .21582 \\
5 & .78072 & .78480 & .99395 & .20914 \\
6 & .78421 & .78142 & .99465 & .21323 \\
7 & .78927 & .78489 & .99532 & .21043 \\
8 & .79399 & .78985 & .99590 & .20604 \\
9 & .79770 & .79446 & .99636 & .20190 \\
10 & .80060 & .79807 & .99673 & .19866 \\
11 & .80319 & .80090 & .99703 & .19613 \\
\hline
\end{tabular}

will contain more than $\left(1+e^{y}\left(2.36 \times 10^{-12}\right)\right) \log ^{7} x>\left(1+4.2 \times 10^{-12}\right) \log ^{7} x$ primes, and other values of $x$ where the interval will have less than $\left(1-e^{y}\left(5.338 \times 10^{-11}\right)\right) \log ^{7} x<\left(1-9.5 \times 10^{-11}\right) \log ^{7} x$ primes (see Figure 4).

Table 2 summarizes some statistics on gaps between zeros and critical points. Our results indicate that $\lambda_{n+1}-\lambda_{n}$ and $c_{n+1}-c_{n}$ increase. We expect that the distance will slowly converge to 1 in both cases. The fact that $c_{n+1}-\lambda_{n}<$ 1 follows from the observation that by (10) $W\left(c_{n+1}\right)=W\left(c_{n+1}-1\right)$, and therefore, since there is a sign change in the interval $\left[c_{n+1}-1, c_{n+1}\right]$, there must be two sign changes.

Added Comment. The editor has brought to our attention the paper, Numerical solution of some classical differential-difference equations, by George Marsaglia, Arif Zaman, and John C.W. Marsaglia, which has since appeared in Math. Comp. 53 (1989), 191-201. In their paper a numerical scheme similar to the one in this paper is used to compute $\omega(u)$ accurately for $u \leq 500$. They also studied other differential-difference equations.

\section{ACKNOWLEDGMENT}

We thank Kevin McCurley for some helpful suggestions.

\section{BIBLIOGRAPHY}

1. A. A. Buchstab, Asymptotic estimates of a general number-theoretic function, Mat. Sb. 44 (1937), 1239-1246. (Russian; German summary)

2. N. G. de Bruijn, On the number of uncancelled elements in the sieve of Eratosthenes, Nederl. Akad. Wetensch. Proc. 53 (1950), 803-812.

3. F. Grupp and H. E. Richert, The functions of the linear sieve, J. Number Theory 22 (1984), 208-239.

4. H. Halberstam and K. F. Roth, Sequences, reprint, Springer-Verlag, New York, Heidelberg, and Berlin, 1983. 
5. Loo-Keng Hua, Estimation of an integral, Sci. Sinica 4 (1951), 393-402.

6. Helmut Maier, Primes in short intervals, Michigan Math. J. 32 (1985), 221-225.

Department of Mathematics, University of California, Davis, California 95616, E-mail: aycheer@ucdavis.edu

Department of Mathematics and Computer Science, San Jose State University, San Jose, CALIFornia 95192.E-mail: goldstn@calstate.bitnet 